\title{
Terpolymerization of 2-ethoxy ethylmethacrylate, styrene and maleic anhydride: determination of the reactivity ratios
}

\author{
C S SANMATHI, S PRASANNAKUMAR ${ }^{\dagger}$ and B S SHERIGARA* \\ Department of Industrial Chemistry, Kuvempu University, Jnana Sahyadri, Shankaraghatta, Shimoga 577 451, India \\ ${ }^{\dagger}$ Fosroc Chemicals (India) Limited, Kuluvanahalli, Bangalore 562 111, India
}

MS received 23 January 2004

\begin{abstract}
Terpolymerization of 2-ethoxyethyl methacrylate (2-EOEMA), styrene (St) and maleic anhydride (Ma) initiated by benzoyl peroxide was carried out in acetone as common solvent for three monomers. The structure and composition of terpolymer were determined by FTIR spectroscopy by recording analytical absorption bands for St $\left(3002 \mathrm{~cm}^{-1}\right)$, Ma $\left(1781 \mathrm{~cm}^{-1}\right)$ and 2-EOEMA $\left(1261 \mathrm{~cm}^{-1}\right)$ units, respectively. The reactivity ratios for the monomers were calculated according to the general copolymerization equations following the Finnemann-Ross and Kelen-Tudos models. The results show that terpolymerization were carried out through primary "complex" mechanism at near-binary copolymerization of [St...Ma] complex with 2-EOEMA. Structure of the resulting terpolymer illustrated by ${ }^{1} \mathrm{H}-\mathrm{NMR}$ and differential scanning calorimeter showed reduction in $T_{\mathrm{g}}$ value.
\end{abstract}

Keywords. Terpolymerization; 2-ethoxyethyl methacrylate; reactivity ratio.

\section{Introduction}

For the last two decades, an acceptor-donor-acceptor (donor) monomer system is an effective method for the synthesis of functional macromolecules with given composition, structure and properties (Triverdi and Culberston 1982; Rzaev 1984). The complex-radical terpolymerization of Ma (acceptor), with vinyl aromatic i.e. St (donor), gives an alternating copolymer, probably through formation of a donor-acceptor complex between St and Ma. Valuable properties can be obtained in terpolymer system in which St along with various functional group monomers such as vinyl acrylate etc (as acceptor or donor), are present besides Ma (Wittmer et al 1969; Ham 1983). This type of terpolymer is applied as water-dispersing agent, paint additive, thickening agent and carrier of biologically active substances etc (Florjanczyle and Krawiec 1933).

As a part of our on going studies on free radical polymerization (Sherigara et al 1999; Rai et al 2000; Yashoda et al 2000, 2001; Prashantha et al 2002), polymeric blends and surfactants (Prashantha et al 2001; Prasannakumar et al 2003; Sanmathi et al 2004), we present here the preliminary data on the radical terpolymerization of the system containing St and Ma complex with 2-ethoxyethyl methacrylate (2-EOEMA). This system was chosen to understand the characteristics of the mechanism of

*Author for correspondence complex radical terpolymerization involving St and Ma. None of these three monomers homopolymerized under the conditions of the reaction selected.

\section{Experimental}

\subsection{Characterization methods}

2.1a Infrared spectra: Infrared spectra of the synthesized terpolymers on $\mathrm{KBr}$ pellets were obtained from Shimadzu FTIR 4200 series spectrophotometer.

2.1b ${ }^{1} H$-NMR spectra: The ${ }^{1} \mathrm{H}-\mathrm{NMR}$ spectra of the terpolymer was recorded on a $90 \mathrm{MHz}$ Varian EM-360 spectrometer at room temperature as $10-15 \mathrm{gl}^{-1}$ solutions, in deuterated chloroform with TMS as internal standard.

2.1c Glass transition temperature: The glass transition temperature was determined on a Mettler TA4000 DSC. Temperature and energy calibrations were carried out with indium. Samples weighing between 12 and $15 \mathrm{mg}$ were used in all cases. The scan rate was $10^{\circ} / \mathrm{min}$ in air. The glass transition temperature $\left(T_{\mathrm{g}}\right)$ was calculated as the inflection point of the jump of heat capacity.

\subsection{Materials}

Styrene and 2-ethoxyethyl methacrylate (Aldrich) were washed with aqueous sodium hydroxide solution and dis- 
tilled water followed by drying over anhydrous calcium chloride and finally distilled under vacuum for further use. Maleic anhydride (Aldrich) was purified before use by recrystallization from anhydrous benzene and sublimation in vacuum. Benzoyl peroxide of analytical grade (S-d fine), was purified by recrystallization in chloroform methanol mixture. The solvents used, diethyl ether and acetone, were of analytical grade (E-Merck).

\subsection{Synthesis}

Synthesis of ternary copolymer with different compositions, were carried out in a $100 \mathrm{ml}$ round bottom flask with acetone as solvent and benzoyl peroxide as initiator at $60^{\circ} \mathrm{C}$. The reaction flask was purged with nitrogen gas continuously and reactions were continued up to $15 \%$ conversion. On completion of reaction, the mixture was cooled to room temperature to terminate polymerization. Cooled mixture was poured into a large volume of diethyl ether to precipitate the terpolymer and was separated by filtration. Obtained terpolymer products were in powder form, which were dried under vacuum at $50^{\circ} \mathrm{C}$ to constant weight (scheme 1).

\section{Results and discussion}

\subsection{Infrared spectroscopy (IR)}

The terpolymers synthesized were characterized by FTIR spectroscopy for the determination of St, Ma and 2-2EOEMA units. The absorption band value for characteris- tics groups are, $3100-3000 \mathrm{~cm}^{-1}(-\mathrm{CH}$ in aromatic fragments), 1225-1200 (asymmetric C-O-C stretching) and 1870-1770 (anhydride groups). Mole fractions (mol\%) of commoners units $\left(m_{1}, m_{2}\right.$ and $\left.m_{3}\right)$ in $\mathrm{Ma}\left(M_{1}\right)-\mathrm{St}-\left(M_{2}\right)-2-$ 2-EOEMA $\left(M_{3}\right)$ terpolymer were obtained by FTIR analysis, and the data were calculated according to the following equations (Rzaev et al 2002).

$$
m_{1}=\frac{A^{1781} / M_{\mathrm{Ma}}}{A^{3002} / M_{\mathrm{St}}+A^{1781} / M_{\mathrm{Ma}}+A^{1261} / M_{2-\text { EOEMA }}} \times 100
$$

$m_{2}=\frac{A^{3002} / M_{\mathrm{St}}}{A^{3002} / M_{\mathrm{St}}+A^{1781} / M_{\mathrm{Ma}}+A^{1261} / M_{2 \text {-EOEMA }}} \times 100$

$m_{3}=\frac{A^{1261} / M_{\mathrm{EOEMA}}}{A^{3002} / M_{\mathrm{St}}+A^{1781} / M_{\mathrm{Ma}}+A^{1261} / M_{2-\mathrm{EOEMA}}} \times 100$

where $A=\log (1 / T), A=$ absorbency and $T=\%$ transmittance.

$M_{\mathrm{Ma}}, M_{\mathrm{St}}$ and $M_{\text {2-EOEMA }}$ are molecular weights of $\mathrm{Ma}, \mathrm{St}$ and 2-EOEMA monomer units, respectively. The composition of terpolymers obtained by FTIR analysis is presented in figure 1 and table 1.

The donor-acceptor interaction between $\mathrm{St}$ and $\mathrm{Ma}$ results in equimolar $(1: 1)$ charge transfer complex (CTC) system and was studied by various methods (Rzaev et al 1984).

$$
\mathrm{Ma}+\mathrm{St} \rightarrow[\mathrm{Ma} \ldots \mathrm{St}]
$$<smiles>C=CC(=O)OC(=O)C=CC(=O)C(C)=CCOCC</smiles>

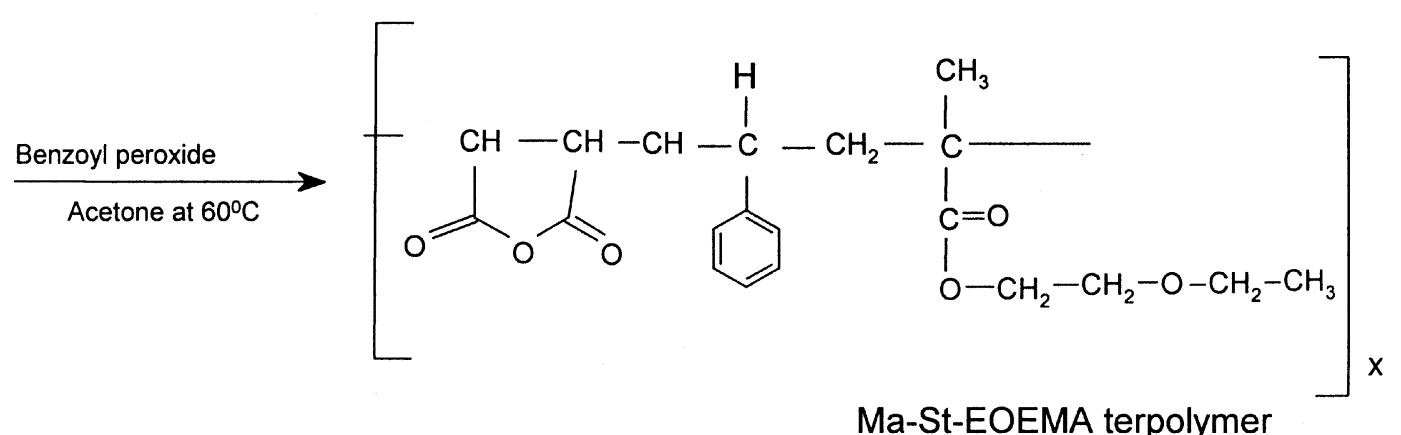

Scheme 1. Preparation of terpolymer reaction. 


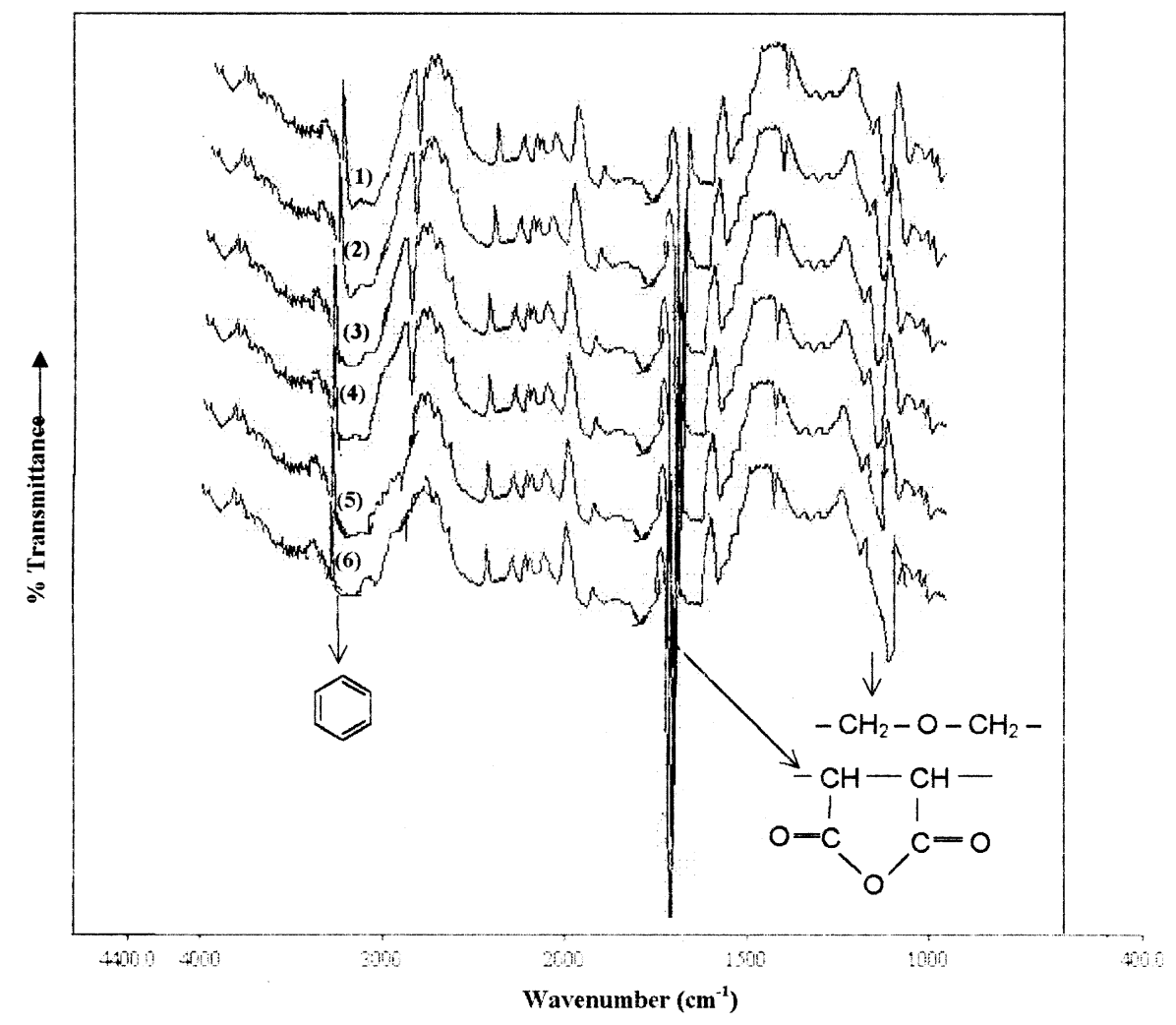

Figure 1. FTIR spectra of Ma-St-2-EOEMA terpolymers prepared in various monomer feeds (Ma: St: 2-EOEMA): (1) $35: 50: 15$, (2) $30: 50: 20$, (3) $35: 35: 30$, (4) $30: 30: 40$, (5) $25: 25: 50$, (6) $20: 20: 60$. (Absorption band value of characteristics groups: $3002 \mathrm{~cm}^{-1}$ (-CH in aromatic fragments); $1261 \mathrm{~cm}^{-1}$ (asymmetric $\mathrm{C}-\mathrm{O}-\mathrm{C}$ stretching); $1781 \mathrm{~cm}^{-1}$ (anhydride groups)).

Table 1. Analysis of the data for the determination of Ma-St-2-EOEMA terpolymer composition synthesized in the various initial monomers mixtures.

\begin{tabular}{lcccccc}
\hline & \multicolumn{3}{c}{ Monomer feed (mol\%) } & & \multicolumn{3}{c}{ Terpolymer composition (mol\%) } \\
\cline { 1 - 2 } \cline { 6 - 7 }$[\mathrm{MA}]$ & {$[\mathrm{St}]$} & {$[2-E O E M A]$} & & {$[\mathrm{MA}]$} & {$[\mathrm{St}]$} & [2-EOEMA] \\
\hline 35 & 50 & 15 & & $28 \cdot 68$ & $35 \cdot 4$ & $35 \cdot 92$ \\
30 & 50 & 20 & & $36 \cdot 2$ & $26 \cdot 4$ & $37 \cdot 4$ \\
35 & 35 & 20 & & $25 \cdot 28$ & $21 \cdot 57$ & $53 \cdot 15$ \\
30 & 30 & 40 & & $28 \cdot 96$ & $17 \cdot 03$ & $51 \cdot 94$ \\
25 & 25 & 50 & & $22 \cdot 89$ & $22 \cdot 01$ & $64 \cdot 1$ \\
20 & 20 & 60 & & $21 \cdot 37$ & $24 \cdot 8$ & $65 \cdot 77$ \\
\hline
\end{tabular}

The CTC formation constant $\left(K_{\mathrm{C}}\right)$ for Ma...St complex was reported earlier by Hanna-Ashbaugh (Hanna et al $1964)$ as $K_{\mathrm{c}}\left(60^{\circ} \mathrm{C}\right)=0.289$ in deuterated chloroform. The CTC effects in radical terpolymerization with Ma and various donor-donor or donor-acceptor vinyl monomers were clearly illustrated (Rzaev et al 1984; Florjanczyle and Krawiec 1989; Yang and Otsa 1992). From these studies it can be suggested that the Ma and St monomer pairs will behave as complexed monomer in radical copolymerization with acrylics. The difference in reactivity of the Ma...St complex in complex radical copolymerization with different acrylic comonomer causes formation of terpolymer with different ratios of 2-EOEMA units.

The ternary monomer systems studied can be classified as acceptor (Ma) and donor (St) and acceptor (2-EOEMA) which can be characterized as follows (Rzaev 1999):

(i) The acceptor-donor monomer pair of Ma and St has a tendency to form the complex. 
(ii) Neither Ma nor St get homopolymerized in selected conditions of terpolymerization.

(iii) The formation of poly 2-EOEMA fragments in low conversion conditions can be ignored by taking into consideration the high reactivity of the macroradicals towards other monomers.

(iv) The formation of 2-EOEMA-St and 2-EOEMA-Ma diad can also take place. While in this case, St and Ma macroradicals have low activity towards 2-EOEMA monomers as compared with $\mathrm{Ma}$ and St monomers, respectively.

On the basis of these selective characteristics of the self organized ternary system studied, the elementary stages of propagation reaction can be proposed for participation of both complexed and free monomers in the following preferable initiation and chain growth reactions (Rzaev 1999):

a. Initiation via complex-bond monomers

$$
\begin{aligned}
& \mathrm{R}^{\bullet}+\mathrm{Ma} \ldots \mathrm{St} \rightarrow \mathrm{R}-\mathrm{Ma}^{\bullet} \ldots \mathrm{St} \rightarrow \mathrm{R}-\mathrm{Ma}-\mathrm{St}{ }^{\bullet} \\
& \mathrm{R}^{\bullet}+\mathrm{St} \ldots \mathrm{Ma} \rightarrow \mathrm{R}^{-\mathrm{St}^{\bullet}} \ldots \mathrm{Ma} \rightarrow \mathrm{R}-\mathrm{St}-\mathrm{Ma}{ }^{\bullet}
\end{aligned}
$$

b. Initiation via free monomers

$$
\begin{aligned}
& \mathrm{R}^{\bullet}+\mathrm{St} \rightarrow \mathrm{R}-\mathrm{St}^{\bullet} \\
& \mathrm{R}^{\bullet}+\mathrm{Ma} \rightarrow \mathrm{R}-\mathrm{Ma}^{\bullet} \\
& \mathrm{R}^{\bullet}+2 \text {-EOEMA } \rightarrow \text { R-2-EOEMA }
\end{aligned}
$$

c. Chain growth via complex-bond monomers

$$
\begin{aligned}
& \sim \mathrm{Ma}^{\bullet}+\text { St...Ma } \rightarrow \text { Ma-St-Ma } \rightarrow \sim \mathrm{Ma}^{\bullet} \\
& \sim \mathrm{St}^{\bullet}+\mathrm{Ma} \ldots \mathrm{St} \rightarrow \mathrm{St}-\mathrm{Ma}-\mathrm{St} \boldsymbol{\mathrm { S }}^{\bullet} \rightarrow \sim \mathrm{St}^{\bullet} \\
& \sim 2 \text { EOEMA }^{\bullet}+\text { St...Ma } \rightarrow \text { 2-EOEMA-St-Ma } \rightarrow \sim \mathrm{Ma}^{\bullet} \\
& \sim \text { 2-EOEMA }{ }^{\bullet}+\text { Ma...St } \rightarrow \text { 2-EOEMA-Ma-St }^{*} \rightarrow \sim \mathrm{St}^{\circ}
\end{aligned}
$$

d. Chain growth via free monomers

$$
\begin{aligned}
& \sim \mathrm{Ma}^{\bullet}+\mathrm{St} \rightarrow \mathrm{St}^{\bullet} \\
& \sim \mathrm{Ma}^{\bullet}+2 \text {-EOEMA } \rightarrow \text { 2-EOEMA } \\
& \sim \mathrm{St}^{\bullet}+\mathrm{Ma}^{\circ} \mathrm{Ma}^{\bullet} \\
& \sim 2 \text {-EOEMA }^{\bullet}+\mathrm{Ma} \rightarrow \mathrm{Ma}^{\bullet} \\
& \sim 2 \text {-EOEMA }^{\bullet}+2 \text {-EOEMA } \rightarrow \text { 2-EOEMA }
\end{aligned}
$$

Monomer-terpolymer composition is given in figure 1 and table 1 . As evident from these data, the content of St and Ma units in the terpolymer having different acceptor monomer ratios are close to equimolar.

The results of FTIR analysis of terpolymer synthesized by using various initial monomer ratios are illustrated in figure 1 and table 1. A diagram of monomer-terpolymer composition is given in figure 2. As evident from these results, the content of St and Ma units in the terpolymer having different acceptor monomer ratios are closest to equimolar. It is also shown that the nature of an acceptor monomer (Ma or 2-EOEMA) as well as the change of monomer composition has strong influence on the character of the diagram point distribution (Rzaev 1999) (figure 2).

These data allow one to determine copolymerization constants (reactivity ratio) $r_{1}, r_{2}, r_{1}\left(K_{\mathrm{c}}\right)^{-1}$ and $r_{2} K_{\mathrm{c}}$ for the St-Ma $\left(M_{1}\right)$ complex and 2-EOEMA $\left(M_{2}\right)$ pairs using the modified terminal model of the Kelen-Tudos (KT) (Kelen 1975) and Fineman-Ross (FR) (Fineman 1950) equation in the following forms.

Fineman-Ross Method:

$$
F(f-1) / f=r_{1} K_{\mathrm{c}} F^{2} / f-r_{2} K_{\mathrm{c}}-1 .
$$

Kelen-Tudos Method:

$$
\begin{aligned}
& \eta=\left[r_{1} K_{\mathrm{c}}+r_{2} K_{\mathrm{c}}^{-1} / \alpha\right] \xi-\left(r_{2} K_{\mathrm{c}}^{-1}\right) / \alpha, \\
& \eta=\left[\left(F^{2} / f\right) /\left(F^{2} / f+\alpha\right)\right], \\
& \alpha=\sqrt{\left(F^{2} / f\right)_{\min }\left(F^{2} / f\right)_{\max }} .
\end{aligned}
$$

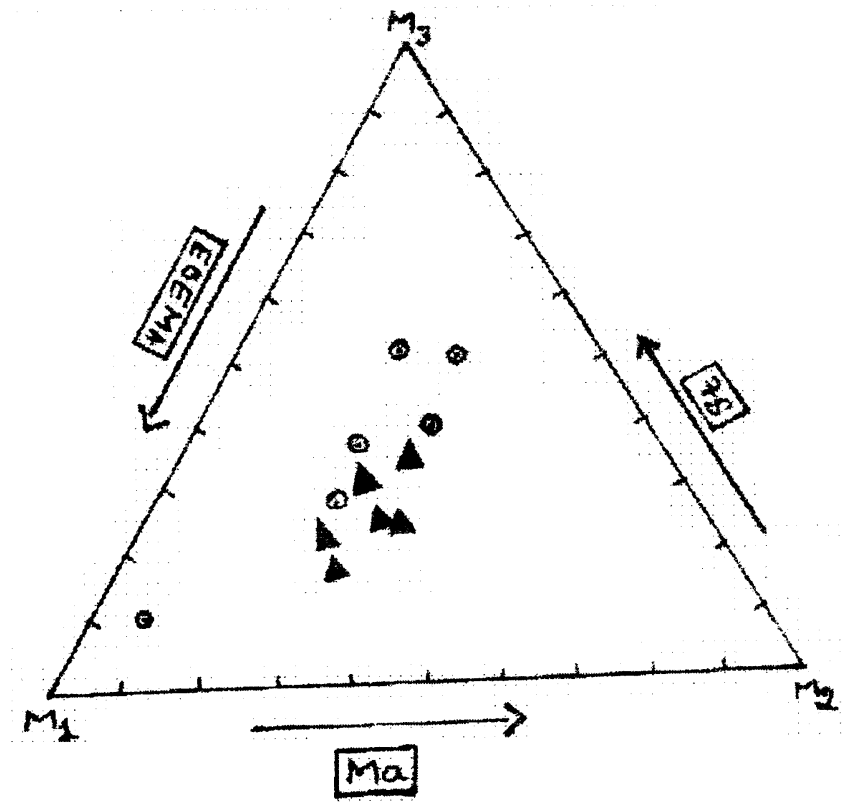

Figure 2. Monomer-terpolymer composition diagram: $(\Theta)$ for monomers, and ( $\boldsymbol{\Delta})$ for composition of St-2-EOEMA-Ma terpolymers. 
Table 2. Experimental data for determining the copolymerization constants $\left(r_{1}\right.$ and $\left.r_{2}\right)$ for MA-St $\left(M_{1}\right)$ and 2-EOEMA $\left(M_{2}\right)$ pair in the low conversion condition.

\begin{tabular}{|c|c|c|c|c|c|c|}
\hline $\begin{array}{l}\text { Monomer ratio } \\
F\left[M_{1}\right] /\left[M_{2}\right]\end{array}$ & $\begin{array}{l}\text { Monomer unit, } \\
\quad f\left(m_{1} / m_{1}\right)\end{array}$ & $F(f-1) / f$ & $F^{2} / f$ & $F^{2} / f+/ \alpha$ & $\eta=\left[\left(F^{2} / f\right) /\left(F^{2} / f+/ \alpha\right)\right]$ & $\xi=\left[F(f-1) / f / F^{2} / f+/ \alpha\right]$ \\
\hline $5 \cdot 667$ & 1.7839 & $2 \cdot 4902$ & $18 \cdot 00$ & $21 \cdot 05$ & $0 \cdot 1182$ & $0 \cdot 854$ \\
\hline $4 \cdot 000$ & 1.673 & $1 \cdot 61$ & $9 \cdot 563$ & $12 \cdot 61$ & $0 \cdot 1276$ & 0.7579 \\
\hline $2 \cdot 333$ & $0 \cdot 8814$ & $-0 \cdot 313$ & $6 \cdot 159$ & $9 \cdot 212$ & -0.0339 & 0.6685 \\
\hline $1 \cdot 500$ & $0 \cdot 8515$ & $-0 \cdot 261$ & $2 \cdot 642$ & 5.695 & $-0 \cdot 0458$ & 0.4639 \\
\hline $1 \cdot 000$ & $0 \cdot 81488$ & $-0 \cdot 227$ & $1 \cdot 227$ & $4 \cdot 28$ & $-0 \cdot 0530$ & $0 \cdot 2866$ \\
\hline 0.667 & $0 \cdot 8577$ & $-0 \cdot 1874$ & 0.518 & $3 \cdot 571$ & -0.0524 & $0 \cdot 14505$ \\
\hline
\end{tabular}

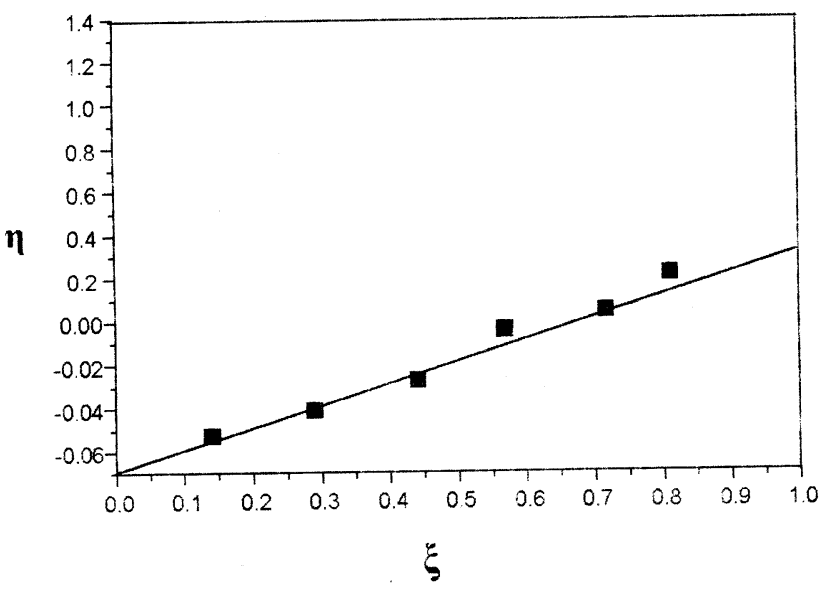

Figure 3. Kelen-Tudos plots for the copolymerization of St...Ma $\left(M_{1}\right)$ with 2-EOEMA $\left(M_{2}\right)$ in acetone at $60^{\circ} . \alpha=$ $r_{1} K_{\mathrm{c}}+r_{2} K_{\mathrm{c}}^{-1} / \alpha$ and intercept $-r_{2} K_{\mathrm{c}}^{-1} / \alpha$.

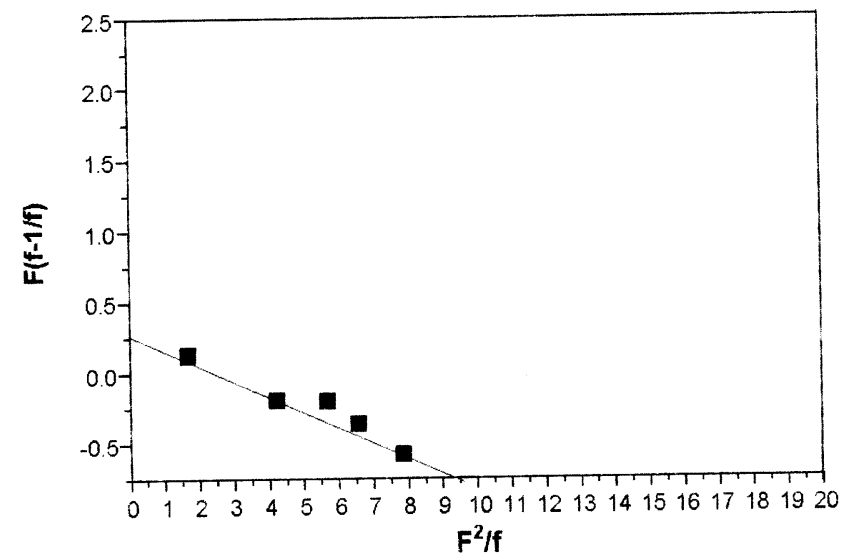

Figure 4. Fineman-Ross plots for the copolymerization of St...Ma $\left(M_{1}\right)$ with 2-EOEMA $\left(M_{2}\right)$ in acetone at $60^{\circ} . \alpha=r_{1} K_{\mathrm{c}}$ and intercept $-r_{2} K_{\mathrm{c}}^{-1}$.

$K_{\mathrm{c}}=$ constant of CTC formation for [St...Ma] complex monomer where

$$
\begin{aligned}
& F=M_{1} / M_{2}=\mathrm{Ma} \ldots \mathrm{St} / 2-\text { EOEMA, } \\
& f=m_{1} / m_{2} .
\end{aligned}
$$

Table 3. Constants of copolymerization for St...MA $\left(M_{1}\right)$ and 2-EOEMA $\left(M_{2}\right)$.

\begin{tabular}{lcc}
\hline Parameter & KT method & FR method \\
\hline$r_{1}$ & $0 \cdot 26$ & $0 \cdot 2$ \\
$r_{2}$ & $0 \cdot 213$ & $0 \cdot 117$ \\
$r_{1} K_{\mathrm{c}}$ & 0.07 & 0.056 \\
$r_{2} K_{\mathrm{c}}^{-1}$ & 0.76 & 0.417 \\
\hline
\end{tabular}

By using (1) and (2) and experimental results and values of the KT and FR equation parameters for the calculation of the monomers reactive ratios, $r_{1}, r_{2}, r_{1} K_{\mathrm{c}}$ and $r_{2} K_{\mathrm{c}}^{-1}$ of the monomers obtained by figures 3 and 4 are presented in tables 2 and 3 .

The effect of observed $K_{\mathrm{c}}$ on the reactivity of the monomers confirms the fact that chain growth proceeds primarily by addition of CTC to growing macroradicals. By these values it can be concluded that [Ma...St] complexes preferentially add to 2-EOEMA comonomer in the St-Ma-2-EOEMA system, and shows that terpolymerization proceeded mainly through a "complex" mechanism in the state of near binary copolymerization of Ma-St and 2-EOEMA comonomer. Only in the chosen ratios of complexed and free monomers the effects of CTC formation from the kinetic point of view allows one to approximate terpolymerization to binary copolymerization of Ma-St $\left(M_{1}\right)$ and 2-EOEMA $\left(M_{2}\right)$.

\section{$3.2{ }^{1} H$-NMR spectroscopy}

The structure of obtained terpolymer (1:1:1 monomers concentration) was illustrated by ${ }^{1} \mathrm{H}-\mathrm{NMR}$ spectroscopy and from ${ }^{1} \mathrm{H}$-NMR spectra (figure 5) characteristics peak signals corresponding to $\delta-0.9$ for $\mathrm{CH}_{3}-, \delta-3 \cdot 8$ for $-\mathrm{CH}_{2}-$ $\mathrm{O}-\mathrm{CH}_{2}-$ in 2-ethoxyethyl methacrylate and $\delta-7 \cdot 3$ for benzene ring of styrene were obtained.

\subsection{Glass transition temperature $\left(T_{g}\right)$}

DSC graph (figure 6) shows $T_{\mathrm{g}}=138^{\circ} \mathrm{C}$ for terpolymer product, which is less than the $T_{\mathrm{g}}$ of styrene maleic anhydride copolymer. 


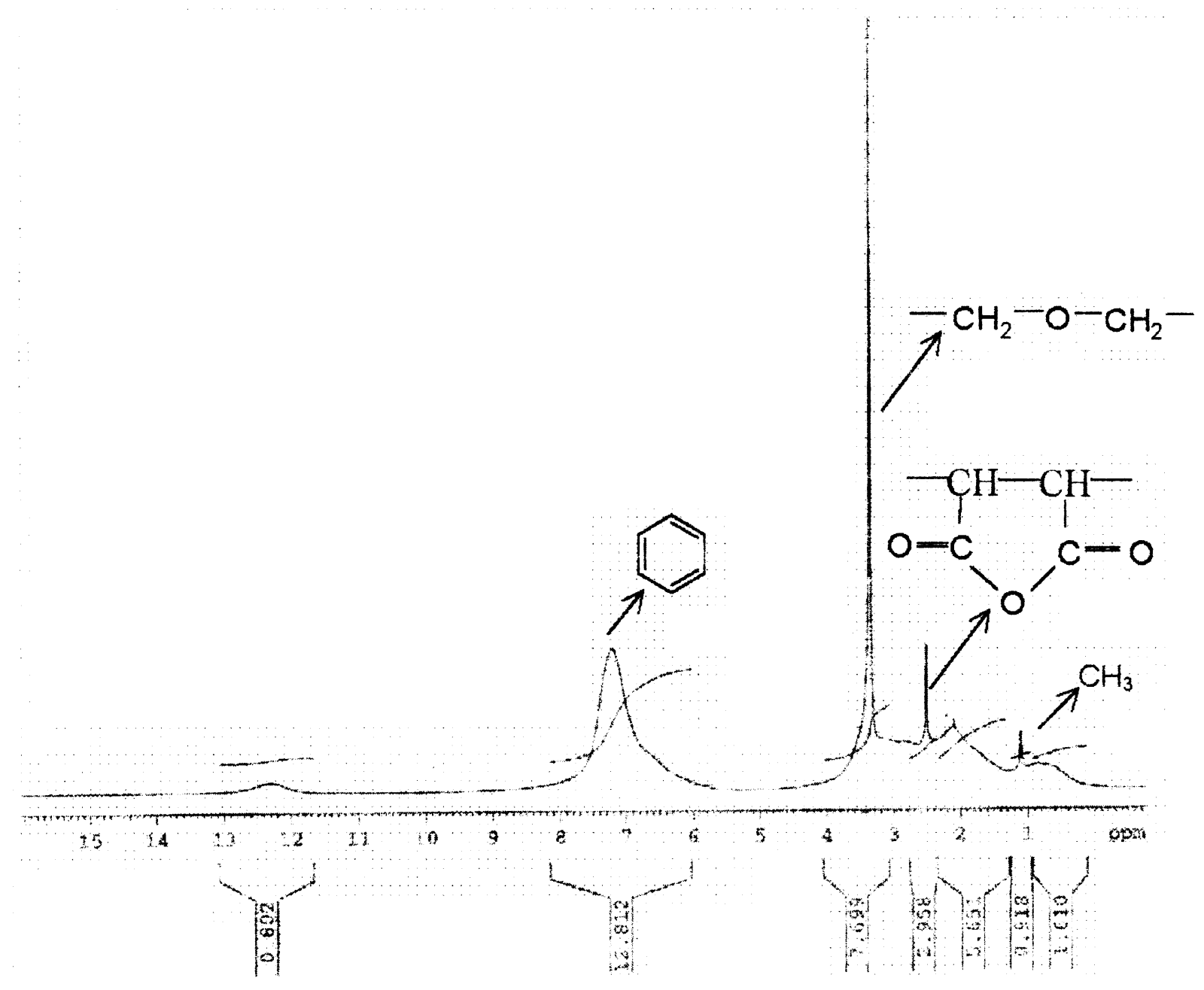

Figure 5. ${ }^{1} \mathrm{H}-\mathrm{NMR}$ spectrum of Ma-St-2-EOEMA terpolymer.

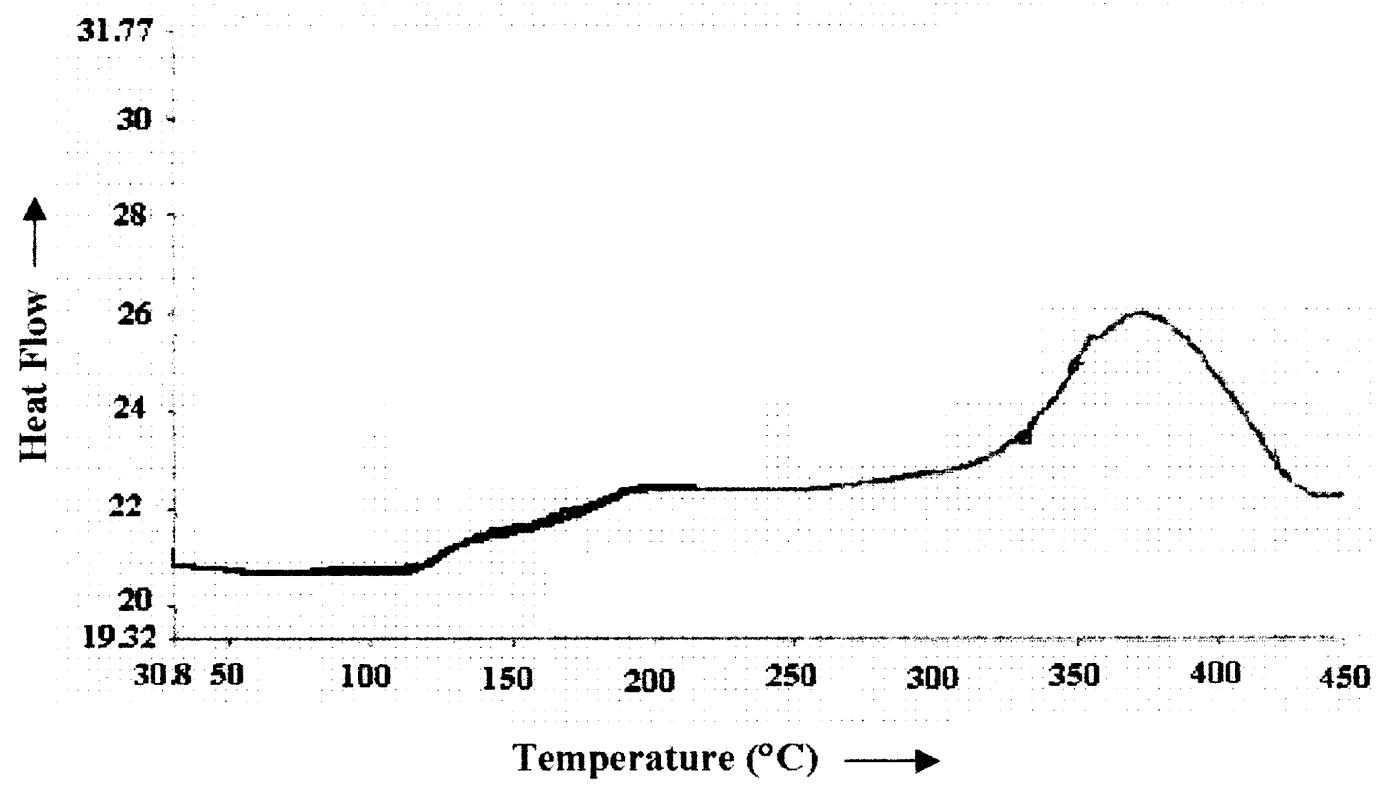

Figure 6. DSC thermograms of Ma-St-2-EOEMA terpolymer, $T_{\mathrm{g}}=138^{\circ} \mathrm{C}$. 


\section{Conclusions}

Calculated reactivity ratio from $\mathrm{KT}$ and FR method $\left(r_{1}<1, r_{2}<1\right)$ clearly indicates that the terpolymer products based on St...Ma-2-EOEMA system has an alternating pattern.

St...Ma-2-EOEMA terpolymer has a $T_{\mathrm{g}}$ of $138^{\circ} \mathrm{C}$ which is much lower compared to that of corresponding St...Ma copolymer i.e. $201^{\circ} \mathrm{C}$ (Hellenne and Bengt 1995). This kind of overall reduction in $T_{\mathrm{g}}$ of the terpolymer may be due to the internal plasticization effect of the 2-EOEMA in the terpolymer product. Obtained reactivity ratio, low $T_{\mathrm{g}}$ value of the terpolymer product and point distribution of three-phase diagram clearly indicates the successful participation of 2-EOEMA as third monomer in terpolymerization process. Further, this type of St...Ma-2EOEMA terpolymer could be a better candidate as surfactant than the corresponding copolymer already in use, in surface science.

\section{Acknowledgement}

One of the authors (CSS) thanks the Kuvempu University, Jnana Sahyadri, for awarding a fellowship.

\section{References}

Florjanczyle Z and Krawiec W 1933 Makromol. Chem. 53189 Florjanczyle Z and Krawiec W 1989 J. Polym. Sci. A; Polym. Chem. 274099

Fineman M and Ross D 1950 J. Polym. Sci. 525

Ham G E 1983 J. Macromol. Sci. Chem. 19693
Hanna M W A and Ashbaugh L 1964 J. Phys. Chem. 68811

Hellene D and Bengt W 1995 J. Polym. Sci. Part A: Polym. Chem. 33571

Kelen T and Tudos F J 1975 Macromol. Sci. Chem. A9 1

Prashantha K, Pai K V and Sherigara B S 2002 J. Appl. Polym. Sci. 84983

Prashantha K, Pai K V, Sherigara B S and Prasannakumar S 2001 Bull. Mater. Sci. 24535

Prasannakumar S, Sanmathi C S and Sherigara B S 2003 Oral presentation at NATCOSEB-XI

Rzaev Z M O 1984 Polymers and copolymers of maleic anhydride (Baku: Elm)

Rai K S, Shivakumara K and Sherigara B S 2000 Eur. Polym. J. 362000

Rzaev Z M 1984 Maleic anhydride polymers and copolymers (Elm: Baku) (in Russian); 1985 Chem. Abstr. 102 11410w

Rzaev Z M, Zeynalov I P, Mamedova S G and Medyakova L V 1984 Polym. Sci. USSR A26 17

Rzaev Z M O, Guner A, Kibare G, Kalplan H C and Asisi A 2002 Eur. Polym. J. 381245

Rzaev Z M O 1999 J. Polym. Sci: Part A: Polym. Chem. 37 1095

Sherigara B S, Yashoda M P, Nayak P V and Padmalatha 1999 J. Phys. Org. Chem. 12605

Sanmathi C S, Prasannakumar S and Sherigara B S $2004 \mathrm{~J}$. Appl. Polym. Sci. (in press)

Triverdi B C and Culberston B M 1982 Maleicanhydride (New York: Plenum Press)

Wittmer R, Hafner F and Gerns H 1969 Makromol. Chem. 104 101

Yashoda M P, Sherigara B S and Venkateshwaran G 2000 Polymer 417381

Yashoda M P, Sherigara B S, Nayak P V and Venkateshwaran G 2001 J. Macromol. Sci: Pure Appl. Sci. 37a 1487

Yang J Z and Otsu T 1992 Macromolecules 25102 